Nano Lett. 2007 December ; 7(12): 3849-3853. doi:10.1021/n1072606s.

\title{
Nanodisk Codes
}

\author{
Lidong Qin ${ }^{\dagger}$, Matthew J. Banholzer ${ }^{\dagger}$, Jill E. Millstone ${ }^{\dagger}$, and Chad A. Mirkin ${ }^{*}$ \\ Department of Chemistry and International Institute for Nanotechnology, Northwestern University, \\ 2145 Sheridan Road, Evanston, IL 60208-3113, USA
}

\begin{abstract}
We report a new encoding system based upon dispersible arrays of nanodisks prepared by OnWire Lithography and functionalized with Raman active chromophores. These nanodisk arrays are encoded both physically (in a "barcode" pattern) and spectroscopically (Raman) along the array. These structures can be used in covert encoding strategies because of their small size or as biological labels with readout by scanning confocal Raman spectroscopy. As proof-of-concept we demonstrate their utility in DNA detection in a multiplexed format at target concentrations as low as $100 \mathrm{fM}$.
\end{abstract}

Encoded materials are used for many applications, including cryptography, computation, brand protection, covert tracking of material goods and personnel, and labeling in biological and chemical diagnostics ${ }^{1-5}$. Often, nanostructures are appealing materials for encoding applications because they can be dispersed or hidden in a variety of media due to their small size, and their chemical and physical properties can be rationally designed in a variety of ways $^{6-9}$. In addition, certain types of nano- and micro-materials are beginning to find application as probes in sensitive and selective molecular diagnostic systems $5,6,9$. These materials include nanoparticles labeled with Raman chromophores ${ }^{10}$, striped nanorods ${ }^{11-13}$, and several types of beads modified with fluorophores ${ }^{5,14-17}$ such as organic dyes and inorganic quantum dots ${ }^{14-17}$. Striped nanorods are a particularly interesting class of materials because they are dispersible entities, allow for massive encoding based upon the length and location of individual chemical blocks within the structures, and can be functionalized using conventional surface chemistries ${ }^{11}$. These nanostructures are typically identified by reflectivity or fluorescence. However, the high degree of overlap between common fluorescent labels, the quenching properties of the metal blocks that comprise the structures, and difficulty in resolving differences in metal reflectivity represent limitations for these systems ${ }^{11,12}$. Herein, we report a new encoding system based upon dispersible arrays of nanodisks prepared by On-Wire Lithography and functionalized with Raman active chromophores. These nanodisk arrays are encoded both physically (in a "barcode" pattern) and spectroscopically (Raman) along the array. These structures can be used in covert encoding strategies because of their small size or as biological labels with readout by scanning Raman spectroscopy.

Recently, we reported a process called On-Wire Lithography (OWL) that allows one to tailor the physical and chemical structure of nanorods to generate a class of nanostructures not accessible by conventional synthetic or lithographic processes ${ }^{18,19}$. Specifically, one can make dispersible, segmented nanorod structures of fixed diameters with gap separations

`chadnano@northwestern.edu.

†These authors contributed equally to this work.

Supporting Information Available. Detailed Methods and materials; assignment of Raman Bands; DNA loading on Au disk surface; calculation of potential NDC structures; calculation of NDC enhancement factor; SEM images of nanorods; additional 3D Raman scans. This material is available free of charge via the internet at http://pubs.acs.org. 
and disk features well-defined along the length of the nanostructure. Gaps between metal sections can be fabricated with dimensions from $2 \mathrm{~nm}$ to many $\mu \mathrm{m}$. This high degree of tailorability has allowed us to probe the relationship between feature size, gap size, and Raman enhancing properties in such a way that we can identify the maximum enhancing structure for a class of disk materials fabricated by OWL ${ }^{19}$. By site isolating disk pairs along the long axis of a nanorod, we can use confocal Raman microscopy (CRM) to independently address each disk pair. Based on the observation that one can create Raman hot spots at specific locations along the long axis of the rod, we hypothesized that one could use disk pair structures that exhibit large enhancements in a novel encoding scheme. Once functionalized with the appropriate chromophores, these nanodisk codes (NDCs), can be identified easily by Raman spectroscopy, and take advantage of the well known surfaceenhanced Raman scattering (SERS) phenomenon ${ }^{10,16,19-23}$. In principle, one can generate a myriad of nanodisk codes simply by varying the number and location of the pairs as well as the type and number of chromophores used as spectroscopic labeling agents. This multilevel approach to encoding nanostructures avoids some of the limitations of striped barcodes by transitioning weaknesses such as fluorescence quenching into advantages in the context of the Raman format. Herein, we report the synthesis, functionalization, and use of NDCs in the context of both physical and nucleic acid labeling.

In a typical experiment, $360 \mathrm{~nm}$-diameter $\mathrm{Au}-\mathrm{Ni}$ nanorods are synthesized by template directed electrochemical synthesis ${ }^{24-26}$, and then converted to disk arrays using OWL methodology (Fig. 1A, Supplementary Fig. S1) ${ }^{18}$. This approach has allowed us to design SERS-optimized Au nanodisk arrays consisting of spatially separated disk pairs and assign a binary coding scheme to these disk pair arrays, where the presence of a disk pair is represented by the number one, and the absence of a disk pair is represented by a zero. For example, if the middle (third) disk pair in a five pair array is intentionally omitted by depositing $\mathrm{Ni}$ in place of an Au disk pair, a code of 11011 is generated. Alternatively, a 10101 barcode is prepared by replacing the second and the fourth Au disk pairs with Ni during the deposition process. With 5 pairs of disks, this encoding scheme, based solely on the location and number of disk pairs, yields 13 distinct nanodisk codes (NDCs) when redundant sequences are eliminated (Fig. 1B). The number of unique coding structures that are available increases exponentially simply by generating longer arrays with more disk pairs (e. g. 25 disk pairs $=2.7 \times 10^{6}$ unique codes, see Supplementary Information for growth calculation and Fig. S2).

As proof-of-concept, we have fabricated three representative NDCs: 11111, 11011, and 10101 codes, (FE-SEM, Supplementary Fig. S1A-C). The Au NDCs 2 are functionalized with a Raman label to yield Raman-active NDCs 3 (Fig. 1A). For the purposes of this study, we have used methylene blue (MB) and para-mercaptoaniline (pMA), two commonly used dyes available from a wide library of Raman chromophores ${ }^{27,28}$. These serve as NDC labels 4 that enable either redundant or complementary detection methods. In this work, these Raman-active molecules are immobilized on the surface of the nanodisk code, where each spot in this array exhibits a SERS enhancement factor of $4.6 \times 10^{8}$ (see Supplementary Information for details of functionalization and calculation) ${ }^{19}$. Note that we have made arrays as long as $12 \mu \mathrm{m}$ thus far, which can support as many as 10 independently addressable disk pairs and 287 disk codes based upon physical encoding alone (see Supplementary Fig. S3).

The Raman-active NDCs 4 were characterized by CRM by integrating the entire spectral intensity from 139 to $2789 \mathrm{~cm}^{-1}$. In this mode, the disk pair features appear as fullyresolved bright spots against a dark, smooth background in both the two- and threedimensional images (Fig. 1C, top, middle). Additionally, a full Raman spectrum is recorded 
at each pixel of the image and provides Raman fingerprint information to accurately assign the labeled NDC (Fig. 1C, bottom).

It is important to note that this approach does not work with the conventional striped metal barcodes, because the disk pairs and optimized gaps are required to achieve high levels of enhanced spectroscopic responses (the optimized architectural parameters for these disk pairs has been determined previously) ${ }^{19}$. To illustrate the importance of the OWL-generated architecture, we prepared an aqueous solution of pre-etched wires (analogous to striped barcodes) and NDCs (made from these pre-etched wires) and simultaneously labeled them with $p$ MA (see Supplementary Information). Both structures were characterized by dark field microscopy and scanning Raman spectroscopy (Fig. 2A, C). Note that each of the five disk pairs of the 11111 NDC exhibit a strong Raman response (Fig. 2A, (right) and 2C (red)) while the unetched structure exhibits almost no signal intensity (Fig. 2A (left) and 2C (blue)). Indeed, the signal intensity of the spectra resulting from the Au disk codes is 142,000 CCD counts higher than the spectra corresponding to the striped wires (calculated by numerically integrating the spectra associated with the locations at the red and blue arrows in Fig. 2A). The ratio of signal intensity between the NDC and unetched wires is approximately 143:1 (normalized for background). To determine if this signal difference was due to differences in surface area, we designed a nanorod structure that contained both a single disk pair and a long Au nanorod segment, where the surface area ratio between the nanorod section and the disk pair is 9:2 (FE-SEM image, Supplementary Fig. S1E). After functionalizing this structure with $p \mathrm{MA}$, we see that the signal intensity from the disk pair is 92 times higher ( 91,000 CCD counts) than the nanorod, despite the much larger surface area and number of chromophores immobilized on the conventional nanorod segment (Fig 2. $\mathrm{B}, \mathrm{D})$.

This binary physical encoding coupled with the high sensitivity Raman readout makes these disk codes attractive as labels in biological tagging schemes. Accordingly, as proof-ofconcept we have investigated the use of NDCs in a three-strand sandwich scheme (Fig. 3A). In these experiments, thiol-modified single-stranded DNA (ssDNA) molecules were attached to the NDC surfaces according to previously published protocols used for gold spherical nanoparticles ${ }^{9}$. These oligonucleotides contained a sequence complementary to one-half of a target ssDNA sequence. The remaining half of the target sequence is complementary to a reporter strand that contains a Raman-active probe. Because the disk pairs generate a SERS-enhancement factor of over 8 orders of magnitude (see Supplementary Information), even a small number of binding events can be detected by CRM (Fig. 1A, 5). In principle, it has an inherent multiplexing capability due to the "barcode" nature of the structures.

To confirm and demonstrate this capability, we employed a three-strand DNA detection system as described above wherein 11011 nanodisk codes are functionalized with an oligonucleotide sequence (called " $\mathrm{NDC}_{11011}$ ") that we designed to be complementary to a section of a DNA target ( $\left.\operatorname{target}_{11011}\right)$. The complement to the second half of this target sequence contained a Cy5 reporter molecule (reporter 11011 ) (Fig. 3A). In a different solution, we functionalized 10101-encoded NDCs with a second sequence ( $\left.\mathrm{NDC}_{10101}\right)$, which is complementary to one half of a second DNA target (target $\left.{ }_{10101}\right)$; the complement to the second half of this target sequence contained a TAMRA reporter molecule (reporter ${ }_{10101}$ ) (Fig. 3A). We then mixed the functionalized 11011 and 10101 NDCs, both target sequences, and both reporter strands in the same solution and allowed the oligonucleotides to hybridize at $37^{\circ} \mathrm{C}$. When both target ${ }_{11011}$ and target $t_{10101}$ are present, positive images are obtained for both 11011 and 10101 disk codes (Fig. 3B). Because the Raman image map contains full Raman spectral information for both Cy5 and TAMRA embedded in every pixel, we were able to specifically monitor the Raman band ranging from 
$525.2 \mathrm{~cm}^{-1}$ to $592.4 \mathrm{~cm}^{-1}$ from Cy5 and apply a filter to the signal output (blue bar, Fig. $3 \mathrm{E})$. By doing so, we have demonstrated that only target ${ }_{11011}$ and reporter ${ }_{11011}$ DNA are present on 11011 disk codes (Fig. 3C). Likewise, we have monitored the Raman band ranging from $1630.0 \mathrm{~cm}^{-1}$ to $1679.0 \mathrm{~cm}^{-1}$ from TAMRA, applied a similar filter (red bar, Fig. 3E), and demonstrated that only target 10101 and reporter ${ }_{10101}$ were present on 10101 disk codes (Fig. 3D). It is important to note that the multiplexing ability of this system is a result of the binary coding architecture and not the different Raman dyes. In this case, two Raman dyes are used simply to illustrate the specificity of the system.

Using the NDCs in a sandwich format, we have detected target DNA at concentrations as low as $5 \mathrm{pM}$ (Fig. 4A). A control experiment containing the same amount of reporter sequences and NDCs, but without target DNA shows no discernable Raman signal (Fig. 4B). Each NDC can be readout within a timescale ranging from seconds to minutes, depending on the desired application (Supplementary Fig. S4). The limit of detection (LOD) is reasonably impressive, considering the assay has not been coupled with a secondary amplification scheme. Even at these low concentrations, the Raman lines (and thus the "fingerprint" character of the spectrum) are still readily observable (Fig. 4C).

Additional sensitivity can be gained if one immobilizes reporter DNA strands on Au nanoparticles $(\mathrm{NP})($ diameter $=13 \mathrm{~nm} \pm 5 \%)$. Using Raman-dye modified AuNPs has the potential to generate a 100-fold increase in signal intensity due to $\sim 100$ additional labeled strands being brought into the Raman hotspot per AuNP binding event. The nanoparticle also quenches the fluorescence of the Raman dye, which has been shown to interfere with Raman scattering. Using the NP-target-NDC sandwich assay 6 (Fig. 1A), a LOD of $100 \mathrm{fM}$ is achievable (Supplementary Fig. S5 and S6). A control experiment where NPs and NDCs are mixed without the presence of a target sequence shows no discernable response, and SEM of the NDCs after mixing confirms that no significant NP binding to the NDCs occurs.

In conclusion, we have developed a novel and potentially powerful encoding system based upon dispersible arrays of nanodisks that are capable of high-sensitivity, multimodal reporting for both chemical and physical covert labeling as well as biomolecule detection. The NDCs are a versatile platform that can be easily tailored using a variety of parameters including chemical label type, disk pair number, and inter-disk pair spacing. These NDCs have the inherent ability to employ labeling chromophores and physical architecture in redundant (where the dye label reporter serves to confirm the binary encoding data), collective (where both the label and the architecture give complementary encoding information), or independent methodologies (where only the label or binary structure is used). This flexibility can be expanded further by synthesizing NDCs with disk pairs of different metal compositions within the same array structure, and functionalizing the different metals with distinct chemical moieties. This additional functionality will enable even more complex encoding structures, where information is stored within the physical architecture and chemical labeling of a single disk array. The use of Raman spectroscopy and the narrow vibrational line signatures of the corresponding dyes (as opposed to the broad emission bands associated with fluorophores), allows one to consider a much larger library of dyes for multiplexing purposes. Finally, although the experiments reported herein are carried out with large, relatively costly instruments, with handheld, conventional Raman spectrophotometers in the marketplace and portable scanning Raman instruments demonstrated in the $1 \mathrm{~b}^{29}$, mobile detection and encoding schemes based on the NDC system are possible.

\section{Supplementary Material}

Refer to Web version on PubMed Central for supplementary material. 


\section{Acknowledgments}

C.A.M. acknowledges the U.S. Air Force Office of Scientific Research (AFSOR), Defense Advanced Research Projects Agency (DARPA), and the National Science Foundation (NSF) for support of this research. C.A.M is also grateful for a NIH Director's Pioneer Award. J. E. M. is grateful to Northwestern University for a Presidential Fellowship. The authors are grateful to Matthew G. Reuter for help on calculating NDC combinatorics.

\section{References}

1. Gershon D. Nature. 2002; 416:885-891. [PubMed: 11976691]

2. Pregibon DC, Toner M, Doyle PS. Science. 2007; 315:1393-1396. [PubMed: 17347435]

3. Nolan JP, Sklar LA. Trends Biotechnol. 2002; 20:9-12. [PubMed: 11742671]

4. Gunderson KL, Kruglyak S, Graige MS, Garcia F, Kermani BG, Zhao C, Che D, Dickinson T, Wickham E, Bierle J, Doucet D, Milewski M, Yang R, Siegmund C, Haas J, Zhou L, Oliphant A, Fan J-B, Barnard S, Chee MS. Genome Res. 2007; 14:870-877. [PubMed: 15078854]

5. Wilson R, Cossins AR, Spiller DG. Angew Chem Int Ed. 2006; 45:6104-6117.

6. Nam JM, Thaxton CS, Mirkin CA. Science. 2003; 301:1884-1886. [PubMed: 14512622]

7. Alivisatos AP. Nat Biotechnol. 2004; 22:47-52. [PubMed: 14704706]

8. Zheng X, Young MA, Lyandres O, Duyne RPV. J Am Chem Soc. 2005; 127(12):4484-4489. [PubMed: 15783231]

9. Rosi NL, Mirkin CA. Chem Rev. 2005; 105:1547-1562. [PubMed: 15826019]

10. Cao YC, Jin R, Mirkin CA. Science. 2002; 297(5586):1536-1540. [PubMed: 12202825]

11. Nicewarner-Peña SR, Freeman G, Reiss BD, He L, Peña DJ, Walton ID, Cromer R, Keating CD, Natan MJ. Science. 2001; 294(5540):137-141. [PubMed: 11588257]

12. Tok JBH, Chuang FYS, Kao MC, Rose KA, Pannu SS, Sha MY, Chakarova G, Penn SG, Dougherty GM. Angew Chem Int Ed. 2006; 45:6900-6904.

13. Stoermer RL, Cederquist KB, McFarland SK, Sha MY, Penn SG, Keating CD. J Am Chem Soc. 2006; 128:16892-16903. [PubMed: 17177440]

14. Eastman PS, et al. Nano Lett. 2006; 6:1059-1064. [PubMed: 16683851]

15. Zanardi P, Rossi F. Phys Rev Lett. 1998; 81:4752-4755.

16. Gunnarsson L, et al. Appl Phys Lett. 2001; 78:802-804.

17. Han M, Gao X, Su JZ, Nie S. Nat Biotechnol. 2001; 19:631-635. [PubMed: 11433273]

18. Qin L, Park S, Huang L, Mirkin CA. Science. 2005; 309:113-115. [PubMed: 15994551]

19. Qin L, Zou S, Xue C, Atkinson A, Schatz GC, Mirkin CA. Proc Natl Acad Sci U S A. 2006; 103(36):13300-13303. [PubMed: 16938832]

20. Orendorff CJ, Gole A, Sau TK, Murphy CJ. Anal Chem. 2005; 77:3261-3266. [PubMed: 15889917]

21. Nie S, Emory SR. Science. 1997; 275(5303):1102-1106. [PubMed: 9027306]

22. Kneipp K, et al. Phys Rev Lett. 1997; 78:1667-1670.

23. Jeanmaire DL, Duyne RPV. J Electroanal Chem. 1977; 84:1-20.

24. Martin CR. Science. 1994; 266:1961-1966. [PubMed: 17836514]

25. Possin GE. Rev Sci Instrum. 1970; 41:772-774.

26. Preston CK, Moskovits MJ. J Phys Chem. 1993; 97:8495-8503.

27. Wang H, Levin CS, Halas NJ. J Am Chem Soc. 2005; 127:14992. [PubMed: 16248615]

28. Fromm DP, Sundaramurthy A, Schuck PJ, Kino G, Moerner WE. Nano Lett. 2004; 4:957-961.

29. Kim J, Liu GL, Lee LP. Optics Express. 2005; 13:4780-4785. [PubMed: 19495396] 


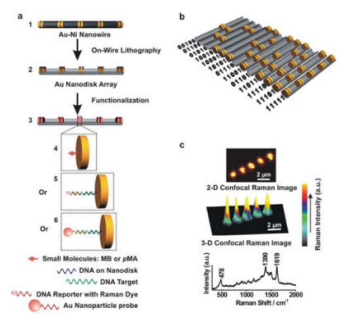

Figure 1.

Scheme of the NDC method. (a) Synthesis and functionalization. (b) 13 possible 5-disk-pair NDCs with corresponding binary codes. (c) Two (top) and three-dimensional (middle) scanning Raman microscopy images of a 11111 NDC. Representative Raman spectrum of methylene blue (bottom) taken from the center of the hot spot generated in the middle disk pair shown in the Raman maps above. 


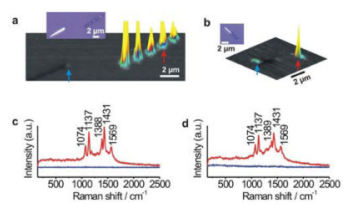

Figure 2.

Comparison of Raman signals in NDCs and conventional striped metal barcodes. (a) Optical (inset) and scanning Raman three-dimensional images of a Au-Ni nanorod (left), a 11111 NDC (right), and (b) an Au nanorod-disk pair array. (c),(d) Raman spectra taken at discrete locations designated by red and blue arrows in (a) and (b), respectively. 


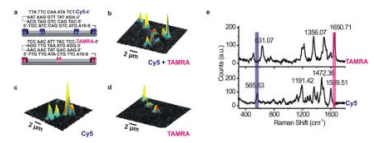

Figure 3.

Multiplexing with NDCs. (a) Scheme of 11011 and 10101 NDC nanostructures showing DNA sequences of target, reporter, and NDC strands. (b) Full-spectrum three-dimensional scanning Raman image of 11011 and 10101 disk codes functionalized with different singlestranded DNA sequences. (c) Filtered Raman image of (b) where the transmitted frequencies are indicated by the blue bar in (e), corresponding to a unique Raman band from the Cy5 reporter $\left(525.2 \mathrm{~cm}^{-1}\right.$ to $\left.592.4 \mathrm{~cm}^{-1}\right)$. (d) Filtered Raman image of (b) where the transmitted frequencies are indicated by the red bar in (a), corresponding to a unique Raman peak from the TAMRA reporter $\left(1630.0 \mathrm{~cm}^{-1}\right.$ to $\left.1679.0 \mathrm{~cm}^{-1}\right)$. (e) Raman spectra of Cy5 and TAMRA from reporter DNA sequences immobilized on NDC after DNA hybridization. N.B. (c, d) are taken with only a fraction of the total Raman signal being collected, the three images in (b-d) do not have the same z-scale, and have been normalized for a straightforward comparison. 
a ATA ACT GAA AGC CAA-Cy $3-5$ '

.'-TAT TGA CTT TCG GTT-3'

'ATG GAT GAT GTG CTA-5'

TAC CTA CTA CAC GAT $\left.\mathrm{A}_{10}-\mathrm{S}\right\urcorner$

II $\mathrm{n} \quad \mathrm{II} \quad \mathrm{II}$-III

b
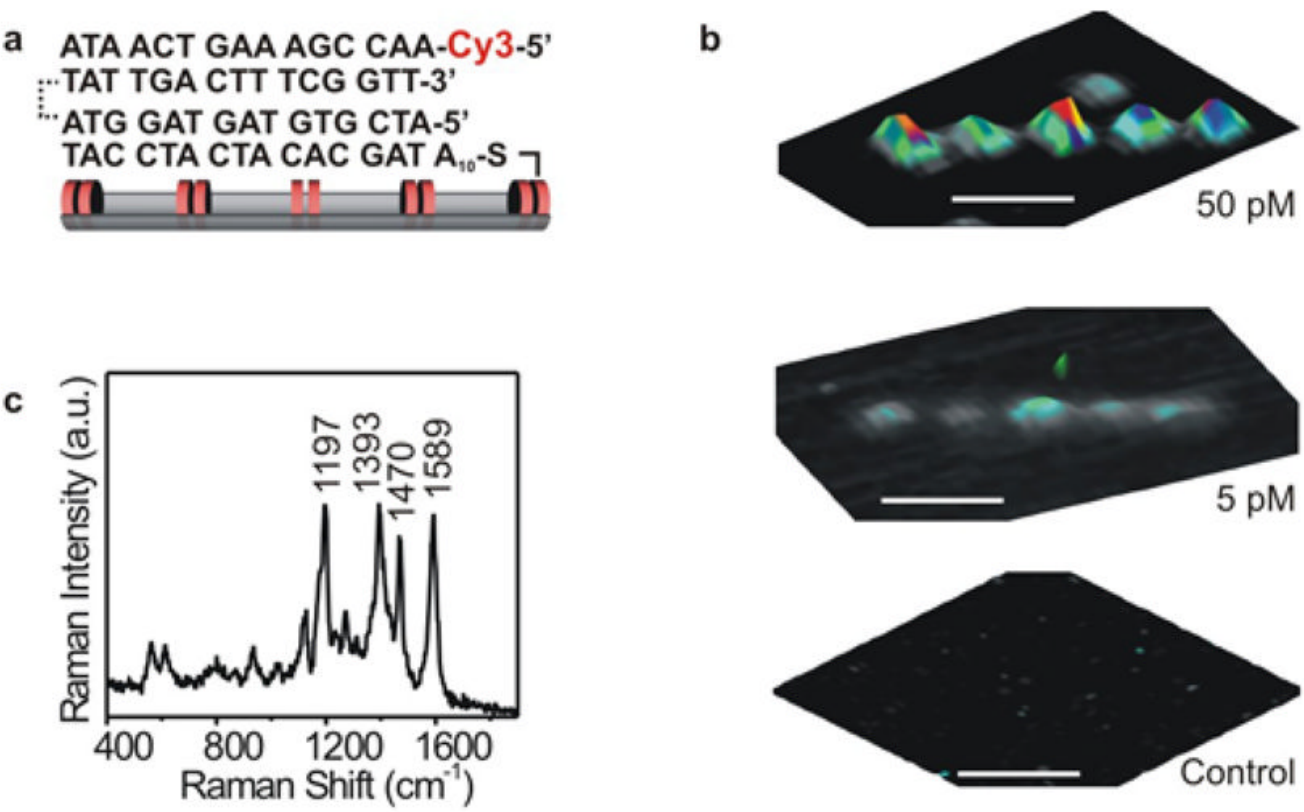

Figure 4.

Three-strand sandwich DNA labeling based on NDC and Raman probe. (a) Schematic representation of three-strand DNA system, including DNA sequences used in these experiments. (b) Three-dimensional scanning Raman images of DNA detection results at target concentrations of $50 \mathrm{pM}, 5 \mathrm{pM}$ and control with no target (top to bottom, respectively). All three images are graphed with the same z-scale. The control experiment (with only reporter and NDC strands, and no target) does not give any readable response. (c) A representative Raman spectrum taken from 3D spectra in (b). 